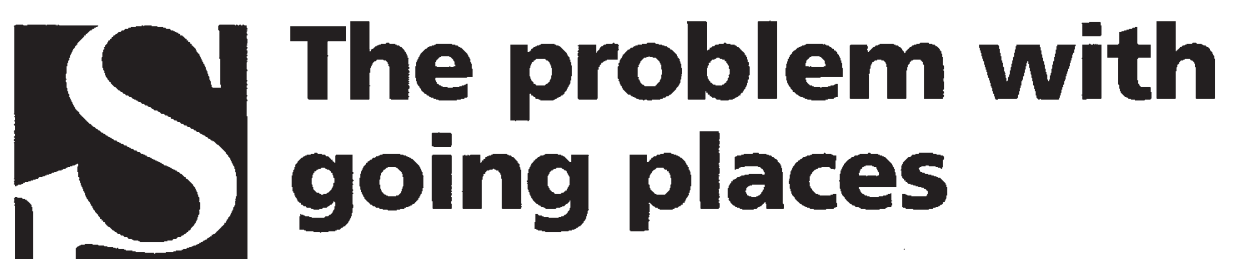

Complex transportation systems demand a high level of organization, and hence energy and information input, to guarantee that the appropriate cargo reaches the appropriate destination-as those who have braved the (apparently) organized chaos of a major international airport will know. Transport of material synthesized within the cell (proteins and carbohydrates) and destined for the exterior or those taken up from the surrounding environment is vitally important for cellular metabolism. Indeed, the complex topology of the membrane-bounded compartments that mediate this transport would put even Denver International airport to shame. The structure of ADP ribosylation factor-1 (ARF-1), which is intimately involved in the regulation of the transfer of materials between these various membrane-bound compartments, has been determined ${ }^{1,2}$ and suggests the means by which the protein helps to police the movement of materials within the cell.

The import, or endocytic, pathway involves both endosomes and lysosomes whereas the export, or biosynthetic-secretory, pathway leads outwards to the surrounding milieu through the endoplasmic reticulum (ER) and Golgi stacks. Material is sorted into and transferred between these various compartments in small, membrane-bound vesicles, which bud from the donor compartment and fuse with the target compartment. The transfer of material is, for the most part, precisely targetted. Clearly, fusion of such vesicles with the first membrane that they encountered would quickly result in the lost of the biochemical identity (and thus function) of the compartments in the cell. The systems responsible for controlling the traffic between these compartments and for maintaining their identity is only now beginning to be revealed ${ }^{3}$.

There are at least two types of coated vesicle that shuttle between compartments within the cell and the surface of the cell, directing the transport of materials from one locale to another. Clathrin-coated vesicles are involved in the selective transport of transmembrane receptors from the trans Golgi and the plasma membrane, whereas coatomer-coated vesicles mediate non-selective transport from the ER and Golgi cisternae. Analysis of the coatomer coat revealed that it consists of eight subunits: seven are structural coat proteins (COPs) and the eighth is ARF.

\section{GTP-binding protein}

ARF is a soluble, monomeric GTP-binding protein that plays a central role in the formation and assembly of coatomer-coated vesicles and the regulation of their transit from donor to target compartments. It also functions to maintain the general integrity of the ER and Golgi by preventing uncontrolled fusion of their membranes. ARF-1's reversible association with membranes is driven by the exchange of GDP for GTP and is mediated by a hydrophobic myristoyl moiety that is cotranslationally attached to the N-terminus of the protein.

The ARF family of proteins is highly conserved-in terms of both structure and function-from yeasts to humans. Both Greasley et al. ${ }^{1}$ and Amor et al. ${ }^{2}$ present the structure of ARF-1, from rat and human respectively. In both cases the inactive, GDP-bound and myristic-acid-free form of ARF-1 was crystallized and the protein consists of a mixed seven-stranded $\beta$-sheet surrounded by six $\alpha$-helices, three on one side of the sheet and three on the other. The core of the ARF-1 structure is similar to that of p21 ras-as expected from the $21 \%$ sequence identity between the two proteins.

ARF, on its own, has little propensity to exchange bound GDP for GTP, it also has no measurable GTPase activity. Rather, it is suggested that exchange and hydrolysis are simulated by an ARF-specific nucleotide exchange factor(s) on the donor compartment membranes and a similarly specific GTPase-activating protein(s) on the target membranes ${ }^{3}$. In this way the 
unidirectional, vectorial transport of coatomer-coated vesicles and their contents is guaranteed, providing one means of maintaining the identity of the donor and target compartments.

The GDP-binding site is located in a cleft whose electrostatic potential is complementary to that of the GDP ligand. The NKXD motif, which is highly conserved in guanine-binding proteins, is involved in binding the guanine base and determining ARF's specificity for guanine nucleotides. The GXXXXGKS/T motif forms part of the'phosphate-binding' loop, and makes an extensive set of interactions with the phosphate groups of the GDP. Nonetheless, the ARF1 equivalent of the putative active-site residue in p 21 ras is displaced by $\sim 5 \AA$ away from the suggested binding site for the $\gamma$-phosphate of GTP and it is suggested that this may be the reason for the lack of intrinsic GTPase activity of ARF-1.

\section{Helices, loops and dimers}

The N-terminal $\alpha$ A helix, the L1 loop that follows it and the $\beta 2_{\mathrm{F}}$ sheet of each monomer are features of the ARF-1 structure which are not seen in p21 ras and are suggested to play essential roles in the biological function of ARF-1 (refs $1,2)$. The myristoyl group is attached to the $\mathrm{N}$ terminal $\alpha A$ helix and there must be a conformational change on nucleotide exchange that makes this group available to interact with the membrane. Amor et al. ${ }^{1}$ point out that L1 appears to be flexible and suggest that, in combination with repeated helical pattern in the $\alpha A$ and $L 1$ sequences, $L 1$ would be able to form a continuation of the $\alpha$ A helix on binding GTP which, in turn, would make the myristoyl group available for membrane insertion.

Both reports of the structure of the ARFGDP complex point out the existence of a noncrystallographic dimer in one of the crystal forms, which may have implications for the vesical transport function of ARF-1. The interface between the two dimers is centred on the $\beta 2_{\mathrm{E}}$ sheet of each monomer, resulting in the formation of a single fourteen-stranded sheet in the dimer. The interface is typical of that seen in many other dimers: the buried surface area is 700-1,000 $\AA^{2}$ and involves mainly hydropho- bic contacts, as well as the hydrogen bonds that form the continuation of the $\beta$-sheet. Nonetheless, there is no evidence that ARF-1 forms a dimer when free in solution, and the protein is monomeric in a second crystal form ${ }^{1}$.

Greasley et al. speculate that dimerization may play a role in the membrane-bound form of ARF-1. The potential close proximity of the ARF-1 monomers when bound to the membrane surface may facilitate their association. Indeed, modelling the myristic acid onto the two $\alpha$ A helices in the dimer shows that the two hydrophobic chains will extend out from the same face of the dimer in a manner that would allow them to interact with the membrane. On the same face of the dimer as the myristoyl moieties there is a patch of positively charged lysine and arginine residues (several from the L1 loop), which would provide a favourable electrostatic interaction with the negatively charged phospholipid head groups of the membrane $e^{1,2}$.

In this model the region equivalent to that in p21 ras which interacts with nucleotide exchange factor is freely accessible, which is consistent with the suggested basis for vectorial vesical transport. Although the dimer may be an artefact of the crystallization procedure, it still remains possible that this hydrophobic patch is involved in mediating protein-protein interactions. Certainly, the equivalent region in p21 ras is the L2 'effector loop' which has been implicated as the region that interacts with effectors and regulators of $\mathrm{p} 21$ ras. If dimerization does occur and does occlude effector binding then the interaction may play an important role in the function of ARF-1.

Unravelling structure of the GTP complex will be the next step in understanding the detailed mechanistic role of ARF-1 in initiating coatomer-coated vesicle assemble. Beyond that, details of the association with the COPs should shed light on vesicle formation, coat disassembly and, perhaps, ARF's role as a membrane-fusion inhibitor.

1. Greasley, S.E. et al. Nature struct. Biol. 2, 797-806 (1995).

2. Amor, J.C., Harrision, D.H., Kahn, R.A. \& Ringe, D. Nature 372, 704-708 (1994).

3. Rothman, J.E. Nature $372,55-63$ (1994).

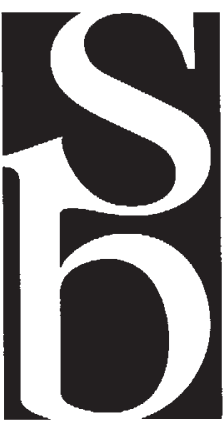

